\title{
La politique européenne d'éducation et de formation
}

\section{Christine Mousny}

\section{OpenEdition}

Journals

Édition électronique

URL : https://journals.openedition.org/ries/1933

DOI : 10.4000/ries. 1933

ISSN : 2261-4265

Éditeur

France Education international

\section{Édition imprimée}

Date de publication : 1 avril 2002

Pagination : 145-151

ISBN : 2-84520-553-8

ISSN : 1254-4590

Référence électronique

Christine Mousny, «La politique européenne d'éducation et de formation », Revue internationale d'éducation de Sèvres [En ligne], 29 | avril 2002, mis en ligne le 25 novembre 2011, consulté le 05 juillet 2021. URL : http://journals.openedition.org/ries/1933; DOI : https://doi.org/10.4000/ries.1933

Ce document a été généré automatiquement le 5 juillet 2021.

(C) Tous droits réservés 


\title{
La politique européenne d'éducation et de formation
}

\author{
Christine Mousny
}

1 Le 24 mars 2000 prenait fin le Conseil européen spécial de Lisbonne, durant lequel les chefs d'États et de gouvernements s'étaient penchés sur une « Europe de l'innovation et de la connaissance ». Leurs conclusions signaient une nouvelle étape, marquante, dans la construction d'une politique européenne d'éducation et de formation. Les États membres s'engageaient sans tambours ni trompettes dans la voie d'une mise en cohérence des systèmes éducatifs, ou tout du moins vers l'acceptation d'un pouvoir supra-national en la matière. Car les enjeux du sommet de Lisbonne pour les systèmes éducatifs des États membres semblent n'avoir pas été véritablement perçus. Peu d'articles en tout cas, et d'ouvrages encore moins, ont été consacrés à cette question depuis. Le Mémorandum sur l'éducation et la formation tout au long de la vie publié le 30 octobre 2000 est la première pierre de ce nouvel édifice, par son thème d'abord qui considère l'ensemble des processus et des dispositifs d'éducation et de formation, par les procédures et les instruments qu'il met en place ensuite, par la complexité de l'organisation politique qu'il instaure enfin.

2 La formation des jeunes et des adultes est depuis 1971 un champ de réflexion et d'action de l'Union européenne, en raison de ses liens évidents avec le secteur économique. C'est seulement en 1993, par le traité de Maastricht, que l'éducation au sens large est entrée dans les attributions de la Communauté. Deux articles y ont été introduits (126 et 127, devenus 149 et 150 en 1999 par le traité d'Amsterdam consolidant le précédent). Ceux-ci invitent la Communauté à encourager l'amélioration de la qualité des systèmes éducatifs, et lui confèrent le droit d'appuyer, voire de compléter, les actions des états membres. Ils donnent également pour rôle à la Communauté de mettre en œuvre une politique de formation professionnelle. Et bien que tenue de respecter la spécificité des systèmes nationaux, l'Union a désormais un pouvoir d'intervention dont elle peut aménager l'étendue et la portée dans une certaine mesure. L'unique véritable interdiction qui lui est faite est d'harmoniser, et par conséquent d'unifier, les systèmes d'éducation et de formation ${ }^{1}$. Désormais, 
l'Europe dispose des moyens d'une véritable politique éducative commune touchant tous les niveaux de formation, de l'éducation pré-élémentaire à la formation des adultes.

3 Certes, elle en a dès lors la possibilité. Pourtant, elle en a peu usé jusqu'à présent. Jusqu'en l'an 2000, son action est restée relativement discrète, peu offensive, ce qui peut s'expliquer par la nécessité de ménager les susceptibilités nationales dans une phase encore fragile de consolidation d'une Europe politique. Il fallait d'abord faire accepter la monnaie unique... Durant cette période, l'action de la Communauté s'est surtout illustrée par :

- la publication du Livre blanc sur l'Éducation et la Formation, "Enseigner et apprendre : vers la société cognitive », en novembre 1995 et par les programmes qui en sont résultés : écoles de la deuxième chance, Erasmus, Leonardo ;

- la publication en 1996 du Livre vert sur les obstacles à la mobilité transnationale, qui a donné lieu aux programmes Socrates et à la recherche de procédés pour la reconnaissance des diplômes ;

- l'adoption en octobre 1996 d'un plan d'action favorisant l'entrée des technologies de l'information dans les écoles, duquel a notamment découlé l'organisation des semaines «netd@ys Europe»; il a été suivi en décembre 1999 par l'adoption d'une communication intitulée « eEurope : une société de l'information pour tous ».

Nombreuses actions il est vrai, mais qui, par leurs objets, n'ont pas vraiment touché le cœur des systèmes éducatifs, c'est-à-dire le noyau de l'enseignement obligatoire, pièce maîtresse des identités nationales. Tout change à l'orée de l'année 2000. Le Portugal, à qui revient alors la présidence de l'Union européenne, propose en janvier un document devant servir de base aux travaux des six mois à venir, intitulé «Emploi, réforme économique et cohésion sociale: pour une Europe de l'innovation et de la connaissance ». Ce document soumet un nouvel objectif stratégique pour la période 2000-2010: «faire de l'Union européenne la zone la plus dynamique et la plus compétitive au monde, un espace fondé sur l'innovation et les connaissances, propre à élever les niveaux de croissance économique et à créer des emplois plus nombreux et meilleurs tout en garantissant une cohésion sociale accrue ». En fait, la présidence portugaise réitère en les appuyant les directives adoptées à partir du traité d'Amsterdam en 1997, notamment pour une coordination des politiques de l'emploi (processus de Luxembourg) et l'impulsion des réformes économiques (processus de Cardiff). Ce qui est nouveau, c'est qu'elle relie intimement les stratégies économiques aux stratégies éducatives, plaçant ainsi l'éducation et la formation au centre des préoccupations européennes. Viviane Reding, commissaire européenne de l'Éducation et de la Culture, soulignait le 20 novembre 2001 dans un discours prononcé à l'Institut royal des Relations internationales que « le Conseil européen de Lisbonne constitue un véritable tournant» et qu'en ouvrant «le grand chantier de «l'Europe de la connaissance ", la recherche, l'innovation, les nouvelles technologies, l'éducation et la formation devenaient « les nouveaux objectifs stratégiques de l'Union. »

5 Ainsi, (c'est à nouveau le document de travail portugais qui parle), «les politiques européennes en matière d'éducation et de formation doivent se projeter au-delà des réformes successives des systèmes existants. Elles doivent avoir pour objectif la construction d'un espace européen d'éducation et de formation tout au long de la vie et l'émergence d'une société cognitive qui offre à chacun sa chance.» Les bases d'une véritable politique éducative commune, globale, cohérente, mais aussi supra-nationale 
et ne se substituant pas en théorie aux politiques nationales, sont offertes à la discussion. Car il s'agit bien de "se projeter au-delà ", et non pas d'agir en plus, en complément de ce qui existe déjà. Il n'est donc pas question d'ingérence, mais d'une vue supérieure, globalisante, au sens économique et politique où on l'entend aujourd'hui. On sent se dessiner de plus en plus clairement au moins deux niveaux décisionnels complémentaires en matière d'éducation: celui des États membres, et celui de l'Union. Structure relativement complexe puisque chacun garde, dans le même temps, son domaine d'action. Il y a donc à la fois stratégies parallèles et stratégies conjointes, complémentarité, égalité et hiérarchie entre les différentes entités.

Impression renforcée par la mission confiée au Conseil Éducation lors du sommet de Lisbonne: à la suite de la présentation du mémorandum, les chefs d'État et de gouvernement ont chargé le Conseil Éducation de mener jusqu'en avril 2001 une réflexion d'envergure sur les objectifs de l'éducation dans l'Union européenne. Le rapport né de ces travaux, intitulé «Les objectifs concrets futurs des systèmes d'éducation » et présenté à Stockholm au printemps 2001, propose une série d'objectifs qui resserrent les liens entre politique communautaire et politiques nationales. «Si les objectifs concrets décrits ci-après ont été formulés à partir des contributions fournies par les États membres, ceux-ci quant à eux envisagent l'avenir dans un cadre d'éducation et de formation tout au long de la vie: il est donc naturel que les deux exercices soient étroitement liés ", est-il argumenté dans le rapport en question².

Sur le fond, pour donner corps aux déclarations du document portugais et afin de poser les bases d'une nouvelle politique d'éducation et de formation, la Commission a élaboré un mémorandum sur l'éducation et la formation tout au long de la vie, dont l'objectif est de lancer un débat dans toute l'Europe sur « une stratégie de mise en œuvre de l'éducation et de la formation tout au long de la vie aux niveaux individuel et institutionnel dans les sphères publique et privée ». Si le sujet n'est pas nouveau, puisque depuis cinq ans les États membres manifestaient régulièrement leur intérêt pour cette question, il n'avait pratiquement pas dépassé le débat d'idées. Les rédacteurs du Mémorandum incitent à l'action, sans ambages : «il est temps de s'atteler à cette tâche $»^{3}$. Il est temps parce que l'éducation et la formation doivent servir une ambition forte, décidée à Lisbonne : «faire de l'Union européenne, d'ici à l'an 2010, l'économie fondée sur la connaissance la plus compétitive et la plus dynamique au monde [...].» Par conséquent, « l'Union européenne doit devenir un exemple à suivre pour le monde entier. L'Europe peut - et doit - montrer qu'il est possible à la fois d'obtenir une croissance économique dynamique et de renforcer la cohésion sociale. $»^{4}$

Pour cela, les rédacteurs estiment que c'est en reconsidérant la politique éducative européenne et les politiques nationales sous l'angle du concept d'éducation et de formation tout au long de la vie que cet objectif pourra au mieux être atteint. Le Mémorandum donne une définition de ce concept fondée sur la stratégie européenne pour l'emploi : il doit être entendu comme «toute activité d'apprentissage utile à caractère permanent visant à améliorer la connaissance, les qualifications et les compétences. (...) La notion d'éducation et de formation tout au long de la vie n'a plus de portée restrictive; il doit désormais s'agir du principe régissant l'offre et la participation, quel que soit le cadre d'apprentissage considéré. $»^{5}$. Puis le mémorandum expose six messages clés (voir encadré) et indique qu'ils peuvent servir de base à un débat ouvert. Plus encore, il engage à la mise en œuvre d'actions, non seulement au niveau de la formation tout au long de la vie (donc de dispositifs post-éducation 
obligatoire), mais également de l'éducation (donc de la formation de base dispensée à tous par des systèmes nationaux d'éducation). Par exemple, le premier message envisage la définition de nouvelles compétences de base, et conséquemment la modification des contenus d'enseignement en fonction de celles-ci: maittrise des technologies de l'information, des langues étrangères, acquisition d'une culture technologique, de l'esprit d'entreprise, d'aptitudes sociales... Il n'est pas interdit de penser qu'on parvienne dans quelques années à l'adoption d'un curriculum de base commun à toute l'Union européenne, lorsqu'on sait qu'une "Charte européenne des compétences fondamentales » a été envisagée à Lisbonne. On pourrait citer aussi le troisième message, qui prévoit notamment la définition d'un rôle nouveau pour les enseignants et formateurs...

9 On est là toujours dans le discours. Mais dans les faits, qu'est-il envisagé pour parvenir à la concertation et, surtout, à la mise en œuvre ? On retrouve là deux niveaux (voire trois) de décision et d'exécution qui s'accommodent du cadre du traité d'Amsterdamex-Maastricht. Au premier niveau, la Communauté impulse, propose un cadrage, des outils. Dans ce sens, l'une des innovations apportées par Lisbonne est l'adoption d'une "méthode ouverte de coordination" qui doit conduire à la concertation entre États membres, puis à la convergence des initiatives. Cette méthode comprend : la définition de lignes directrices européennes ; l'élaboration d'un calendrier d'action pour atteindre les objectifs fixés; l'utilisation si nécessaire d'indicateurs et de références de «bonnes pratiques». Tout cela est déjà mis en pratique. Pour les indicateurs par exemple, les experts ont poursuivi et élargi des travaux initiés en janvier 2000 dans le cadre du rapport sur l'enseignement multimédia, par lesquels ils cherchent à définir des indicateurs adéquats à la définition de l'éducation et de la formation tout au long de la vie. De même pour ce qui concerne les références de «bonnes pratiques»: le Mémorandum en cite douze, pour la plupart européennes (nationales ou communautaires), mais aussi australienne, canadienne, américaine. La Communauté doit aussi ultérieurement réorienter. Son rôle de contrôle paraît renforcé avec la nouvelle méthode adoptée. Cette fois, en effet, tout un dispositif de suivi a été mis en place, avec consultation dans chaque État membre de tous les partenaires impliqués, enquête à mi-parcours recensant les pratiques nationales, rapport en novembre 2001 (un an après l'enclenchement du processus) à partir des résultats de la concertation et recentrant les objectifs. La Communauté semble bien être le pilote.

$10 \mathrm{Au}$ deuxième niveau, les États exécutent les premières directives et proposent des alternatives. Le Mémorandum insiste bien sur le fait que « le changement ne peut venir que des États membres et grâce à leur élan »". Le document précise en outre que « il appartient aux États membres, en tant que responsables des systèmes d'éducation et de formation, de diriger ce débat. Celui-ci doit en outre être dirigé au niveau national et non pas au niveau européen. $\gg^{7}$. Il y a donc, d'abord, une injonction forte pour que des actions soient enclenchées dans les États membres, avant qu'eux-mêmes ne prennent le relais. Ceci pour deux raisons : la première parce que la Communauté n'a pas légitimité à intervenir directement sur les systèmes éducatifs nationaux, et la deuxième parce que l'adhésion des citoyens des États membres doit être recueillie pour une efficacité maximale de la politique engagée.

11 Enfin, on peut déceler un troisième niveau que l'on pourrait appeler, selon la formule aujourd'hui consacrée mais qui demanderait à être clarifiée, le local. Tous les systèmes éducatifs des États membres de la Communauté européenne sont aujourd'hui 
décentralisés, bien qu'à des degrés divers. Les régions, les communes, les établissements d'enseignement, mais aussi les entreprises puisqu'il s'agir de formation tout au long de la vie, sont impliqués tant dans la concertation que dans la proposition d'initiatives et la mise en œuvre de dispositifs. C'est donc non seulement une force de proposition et d'innovation, mais aussi un niveau de décision et d'exécution non négligeable, qui tend à prendre de plus en plus d'importance et qu'il importe de prendre en compte dans l'évolution du champ de la politique éducative européenne.

Il est indéniable que l'expérience de l'Union européenne entraîne les systèmes éducatifs nationaux de la vieille Europe vers une évolution remarquable, si ce n'est vers une révolution. Car c'est bien leur caractère national qui est remis en question, avec tout ce qu'il porte d'identité, de culture, de valeurs. Nous n'entrerons pas dans un débat pour ou contre l'Europe, qui n'aurait ici aucun intérêt. Ce qui est digne d'attention, ce sont les changements qui sont à l'œuvre et la façon dont nous pouvons les recevoir, les accompagner et y prendre part, en fonction de ce que nous sommes et de ce que nous souhaitons pour l'avenir de nos sociétés et des hommes qui les composent. L'Europe ne doit pas forcément nous déranger. Mais elle doit obligatoirement nous interroger.

Les six messages clés du Mémorandum :

\section{Premier message : de nouvelles compétences pour tous}

Objectif : garantir un accès universel et permanent à l'éducation et à la formation afin de permettre l'acquisition et le renouvellement des compétences nécessaires à une participation durable à la société de la connaissance.

Définition de ces nouvelles compétences : « celles indispensables à la participation active dans la société et l'économie de la connaissance » (par exemple la culture numérique, les langues étrangères, des compétences sociales...).

\section{Deuxième message : un investissement accru dans les ressources humaines}

Objectif : parvenir à un accroissement visible de l'investissement dans les ressources humaines afin de placer la priorité sur l'atout majeur de l'Europe - sa population.

\section{Troisième message : l'innovation dans l'éducation et la formation}

Objectif : élaborer des méthodes efficaces d'enseignement et d'apprentissage pour l'offre ininterrompue d'éducation et de formation tout au long de la vie et dans tous les domaines de la vie.

\section{Quatrième message : valoriser la formation}

Objectif : parvenir à une amélioration significative de la façon dont sont perçus et appréciés la fréquentation et les résultats des actions de formation, notamment dans le cadre de l'éducation non formelle et informelle.

\section{Cinquième message : une nouvelle conception de l'orientation}

Objectif : veiller à ce que tout un chacun ait facilement accès à une information et des conseils de qualité sur l'offre de formation dans toute l'Europe, tout au long de sa vie.

\section{Sixième message : rapprocher l'éducation et la formation du niveau local}

Objectif : organiser l'éducation et la formation tout au long de la vie aussi près que 
possible des apprenants, dans leur communauté et, si nécessaire, au sein de structures exploitant les NTIC.

\section{Pour en savoir plus :}

\# Le centre de ressources Sources d'Europe, Grande Arche de la Défense, Paris (accès libre du lundi au vendredi de $10 \mathrm{~h} 00$ à $18 \mathrm{~h} 00$ )

\# Le réseau Eurydice sur Internet : http://www.eurydice.org

\# Les résultats de l'enquête européenne menée par Eurydice et le Cedefop : «Initiatives nationales en faveur de l'éducation et la formation tout au long de la vie », Commission européenne, Éducation et culture, Socrates, mai 2001.

tout au long de la vie, accessible sur le site $\mathrm{du}$ ministère délégué à l'enseignement professionnel: http:// www.enseignement-professionnel.gouv.fr/dernier_vif_4.htm

\section{NOTES}

1. En effet, en raison des termes du traité d'Amsterdam (ex-traité de Maastricht), aucune décision, aucune résolution, aucun texte ne peut viser explicitement l'harmonisation des systèmes éducatifs. Bien souvent, depuis Lisbonne, la disposition protégeant la souveraineté des États en matière d'éducation est contournée, comme dans le discours du ministre de l'éducation portugais, Guilherme de Oliveira Martins, au Conseil Éducation du 8 juin 2000. À propos de l'identification des objectifs concrets futurs des systèmes éducatifs, il déclarait qu'il s'agissait de «coopérer pour échanger les meilleures pratiques afin de réaliser les objectifs identifiés à Lisbonne, en vue d'aboutir non pas à une harmonisation des systèmes éducatifs, mais à leur convergence ».

2. Rapport de la Commission, Les Objectifs concrets futurs des systèmes d'éducation, Bruxelles, 31 janvier 2001, p. 6.

3. Mémorandum sur l'éducation et la formation tout au long de la vie, document de travail des services de la Commission des Communautés européennes, Bruxelles, le 30/10/2000, SEC (2000) 1832, p. 7.

4. Mémorandum, p. 6.

5. Mémorandum, p. 3.

6. Mémorandum, p. 6.

7. Mémorandum, p. 3. 
INDEX

Mots-clés : politique éducative, Union européenne

Index géographique : Europe

\section{AUTEUR}

\section{CHRISTINE MOUSNY}

Christine Mousny, chargée de programme au département Coopération en éducation du CIEP. 